\title{
Germanica
}

\section{Marica Bodrožić, L'un et le multiple}

Marica Bodrožić, The One and the Many

Marica Bodrožić, Einheit in der Vielfalt

\section{Marie-Hélène Quéval}

\section{OpenEdition}

\section{Journals}

Édition électronique

URL : http://journals.openedition.org/germanica/1994

DOI : 10.4000/germanica.1994

ISSN : 2107-0784

\section{Éditeur}

Université de Lille

\section{Édition imprimée}

Date de publication : 31 décembre 2012

Pagination : 51-87

ISBN : 9782913857308

ISSN : 0984-2632

\section{Référence électronique}

Marie-Hélène Quéval, « Marica Bodrožić, L'un et le multiple », Germanica [En ligne], 51 | 2012, mis en ligne le 14 janvier 2013, consulté le 06 octobre 2020. URL : http://journals.openedition.org/germanica/ 1994 ; DOI : https://doi.org/10.4000/germanica.1994

Ce document a été généré automatiquement le 6 octobre 2020.

(c) Tous droits réservés 


\title{
Marica Bodrožić, L'un et le multiple
}

\author{
Marica Bodrožić, The One and the Many \\ Marica Bodrožić, Einheit in der Vielfalt
}

Marie-Hélène Quéval

1 Originaire de Croatie, Marica Bodrožić avait à peine dix ans lorsqu'en 1983, elle quitta son pays natal pour rejoindre ses parents en Allemagne. Dans ses écrits autobiographiques tels que "Sterne erben, Sterne färben... », elle évoque le traumatisme lié à la perte du langage et montre comment la conquête de la nouvelle identité passa par celle des mots. Car c'est par les mots que l'enfant a appris peu à peu à s'orienter dans un environnement inconnu, chaque mot nouveau ouvrant des portes sur des espaces inexplorés, signifiant la découverte de terres vierges, la conquête de nouveaux territoires... L'allemand qui signifiait le renoncement à l'identité originelle de l'enfant, donna ainsi naissance à une identité nouvelle associant harmonieusement l'imaginaire et la sensibilité méditerranéenne à l'exigence de rigueur imposée par l'apprentissage d'une langue étrangère. L'enfance libre et vagabonde dans les paysages embaumés par le thym et le romarin, écrasés de soleil, entourés par le bleu lumineux de la mer et du ciel avait pris fin. Le temps littéraire lui succéda. Marica Bodrožić ne se sert pas de l'allemand pour écrire, c'est au contraire l'allemand qui a imprégné sa sensibilité, formé l'écrivain en elle et qui a élevé une barrière protectrice contre la barbarie des années 1990, la protégeant de la nostalgie, du sentimentalisme facile encouragé par le serbo-croate.

2 Titulaire du prix Chamisso, créé par Harald Weinrich pour encourager les jeunes écrivains allemands d'origine étrangère, Bodrožić avoue son admiration pour un auteur dont elle partage la sensibilité, le goût pour la nature, les paysages, mais aussi les angoisses métaphysiques. Comme celui de Peter Schlehmil, son style plonge ses racines dans l'univers trouble et magique du rêve et du conte de fée. Schlehmil n'a-t-il pas renoncé à son ombre, un double illusoire du moi, pour parcourir le globe terrestre avec ses bottes de sept lieues et en découvrir les secrets? L'ombre qu'il traînait derrière lui comme un poids mort ne l'empêchait-elle point de s'ouvrir à l'infinité du monde ? C'est en renonçant à cette identité unique et limitée que Schlehmil s'ouvre à la multiplicité des cultures. Chamisso lui aussi a survécu aux horreurs de la guerre civile, si l'on 
accepte de donner ce nom à la Révolution française. Certes, Marica Bodrožić n'a pas fui la guerre, celle-ci l'a surprise alors qu'elle séjournait en Allemagne depuis longtemps déjà. Mais elle partage avec lui la tristesse de voir le pays de son enfance dévasté par la barbarie. Elle-même est heureuse de reconnaître certaines similitudes entre son destin et celui du poète romantique. Comme lui, elle a dû renoncer à sa langue d'origine pour adopter l'allemand. Comme lui, elle a commencé par imaginer des contes, se réfugiant dans un monde dédié à la beauté partout visible malgré les dictatures et les conflits. Si les premiers écrits rassemblent surtout des poèmes, en vers libres ou en prose rythmée, ou encore des contes philosophiques idéalisant les paysages croates $^{1}$, les romans intègrent mieux la réalité politique et psychologique. Ses deux derniers romans Das Geheimnis der Libellen ${ }^{2}$ et Kirschholz und alte Gefühle ${ }^{3}$ en particulier situent leur action pendant la guerre qui secoua la Yougoslavie dans les années 1990. Ils forment un tout, chacun donnant la parole à une des deux amies Arjeta et Nadeshda, ce qui permet à l'auteur de présenter la même période selon deux perspectives subjectives différentes.

3 Marica Bodrožić accepte ainsi plutôt mal d'être considérée comme représentante d'une littérature différente, "mineure », aurait dit Gilles Deleuze, une littérature de l'exil. Elle ne veut pas servir d'exemple pour une "intégration " réussie, ne veut pas être objet d'une étude sociologique. La question de l'apparence nationale ne se pose pas en ces termes. Étrangère, certes, elle l'a été et l'est toujours un peu... Mais l'exil est plutôt métaphysique... l'homme ne se sent jamais vraiment chez lui quelque soit le lieu ou le pays qui l'accueille ou l'a vu naître... Loin de se résumer au changement d'État et de nation, cette instabilité identitaire caractérise la condition humaine.

4 La véritable patrie de l'écrivain reste la langue. Chez elle, tout se passe simultanément. Les deux idiomes, les deux identités coexistent harmonieusement, s'enrichissant mutuellement en donnant naissance à une écriture différente par ses thématiques et une façon de ciseler la langue parfois même jusqu'au maniérisme. Marica Bodrožić dit ressentir un amour sensuel et profond pour cette langue paradoxale qu'elle trouve si claire, si lumineuse, si riche et si souple, et qui a signifié pour elle une source de libération malgré la déformation du national-socialisme. Elle sait que certains mots trop chargés d'histoire doivent être évités, comme on évite de toucher une blessure non encore cicatrisée. Le premier contact a été musical... l'enfant était fasciné par les sons, les harmonies qui s'en dégageaient, avant même de comprendre intellectuellement, par sa raison, le «sens » qui se cachait derrière... Peu à peu, de la masse, certes mélodieuse mais informe des sons, s'est dégagé un sens, une couleur, un parfum, une tradition, une histoire parfois conflictuelle et tragique... Ce fut un apprentissage charnel permettant l'élargissement de la conscience tout autant que le raffinement de la sensibilité.

\section{La diaspora et le rêve de l'identité multiple}

L'expérience de l'altérité lui a enfin permis de se trouver tout à la fois unique et plurielle. Plus qu'une double identité nationale, Bodrožić incarne la nouvelle condition des Européens qui apprennent peu à peu à se libérer du modèle du xixe siècle, un modèle fort ébranlé au cours du siècle suivant, non seulement par les guerres qui ont paradoxalement œuvré au rapprochement des peuples mais aussi par la volonté politique. L'Europe, la naissance et le renforcement d'une identité européenne sont ainsi devenus le but affiché et souvent aussi sincère des jeunes générations. Pourtant, il 
ne suffit pas d'abolir les frontières et de renforcer la coopération économique des États pour créer une identité européenne ressentie par une grande majorité de citoyens comme subsidiaire. Marica Bodrožić fait partie de ceux pour qui, dit-elle, l'Europe est devenue une «idée fixe ». La guerre de Yougoslavie est à ses yeux un abcès, une crise d'identité particulièrement dévastatrice qu'on aurait tort de penser hors du contexte historique et des conflits qui ont troublé le xxe siècle. Quand elle pose la question des identités nationales, c'est pour démontrer l'absurdité du politique qui ignore la volonté des peuples. Cette guerre signifie à ses yeux un véritable assassinat de la Yougoslavie, et avec elle celui de l'utopie de l'état pluriethnique. Elle exprime le refus de la multiplicité, de la pluralité ; or la Yougoslavie, c'était la pluralité dans l'unité.

Les personnages de ses romans ont toujours des origines multiples ${ }^{4}$, juives, croates et bosniaques, sinon aussi russes et allemandes à la fois. Et ce n'est sans doute pas par hasard qu'elle a traduit le roman d'Igor Stickš, Die Archive der Nacht ${ }^{5}$, où le narrateur qui se croit autrichien, découvre son ascendance juive et bosniaque. Dans ce roman, on retrouve des problématiques qui lui sont chères : l'identité multiple, l'absurdité de la guerre civile, la complexité des Balkans et de leurs divers peuples, mais aussi la quête perdue d'avance du moi, la passion destructrice. En référence à Max Frisch, Igor Stickš pose la question de l'identité, de l'emprisonnement dans une personnalité imposée, de la fuite hors de soi.

7 Tous ces thèmes se retrouvent dans Kirchholz où tous les personnages sont plus ou moins en attente, en fuite ou en quête d'eux-mêmes. Tous se rencontrent fortuitement à Paris, titulaires de bourses d'études, ou en instance de départ pour d'autres horizons, cherchant à échapper à un passé cruel, comme celui qui frappe la Bosnie... Arjeta, la narratrice aux origines kosovare, serbe, croate et bosniaque à la fois, sans oublier le grand-père issu de la Bucovine roumaine, est originaire de Sarajevo, une ville qu'elle a quittée sous les bombes. La guerre condamne les hommes à l'errance et à l'exil. Arjeta évoque son séjour à Paris et sa fuite à Berlin. Étudiante en philosophie, elle obtient une bourse comme réfugiée politique, bien que n'ayant pas demandé ce statut. Tout en refusant de se poser en victime, elle a vécu l'horreur, en la personne de ses frères jumeaux déchiquetés par une grenade, mort absurde à l'image de cette rage insensée. La guerre constitue sinon le sujet, du moins le cadre du récit, tous les personnages étant plus ou moins engagés, en tant que témoin, acteur ou observateur : Arik gagne sa vie en la photographiant, la mère de la narratrice après avoir été privée de ses deux fils et de son mari, témoigne, impuissante, mais refuse l'exil qu'elle assimile à une désertion, l'oncle, vétéran de la Seconde Guerre mondiale, héros communiste, vit banni à Paris par Tito, l'amie slovène attend, son billet d'avion en poche, le jour fixé pour son départ aux USA. Au hasard des rencontres, dans le restaurant de Sophie à la Bastille, la diaspora yougoslave se réunit avant de reprendre la route vers des destins plus cléments, loin de la barbarie. Toutes ces vies se croisent pour un temps très court puis se détachent peu à peu les unes des autres...

\section{L'idéologie destructrice}

8 Certains restés au pays, croient pouvoir remodeler la langue et avec elle la conscience nationale d'un peuple qui jusque-là semblait fort bien s'accommoder de sa diversité, comme le petit Mateo devenu grand. Ce personnage plutôt falot et lâche avant la guerre se découvre une âme de héros, méprisant pour les larmes de ses camarades de combat, 
insensible à leurs souffrances, indifférent aux morts inutiles. Le roman montre l'absurdité d'un conflit armé qui, en trahissant les valeurs humanistes, détruit ce qu'il prétend reconquérir. Là aussi, la conquête identitaire passe par la langue ${ }^{6}$, mais cette purification du langage prend des allures grotesques comme lorsqu'on remplace le mot d'origine latine «avion » par celui de «Luftdurchsegelungsgerät». La langue perd sa fonction originelle, libératrice, et se transforme en une prison ${ }^{7}$. Le serbo-croate cesse d'exister pour laisser place à un nouveau langage, celui de la dictature, des processions au flambeau, des ordres stupides, du culte de la nation, de l'obscurantisme des faux révolutionnaires. Le style habituellement doux s'enflamme pour exprimer une juste colère face à la folie de ce nationalisme destructeur.

9 Car on n'impose pas la nation par le feu et la guerre! La ferveur patriotique n'excuse pas le crime. Une nation construite sur de telles monstruosités ne mérite aucun respect. Le personnage de Mateo prend donc une signification toute particulière, car il incarne la folie meurtrière des années 1990, l'aveuglement et le fanatisme, le suicide enfin de la Yougoslavie. On ne crée pas une conscience nationale en détruisant les monuments, en niant une histoire qui, non sans raison, a été ressentie comme traumatisante, mais qui reste malgré tout celle de ce pays. La juste révolte contre une dictature honnie ne peut s'exprimer dans la destruction des monuments antifascistes ou par des insultes à la mémoire de Tito. Et cette nouvelle « nation ", qu'elle soit croate, serbe ou bosniaque, trahit ses propres valeurs, bien plus encore que la Yougoslavie dans sa diversité n'avait pu le faire.

Car ce n'est pas en faisant œuvre de destruction qu'on peut construire une société nouvelle et ce n'est pas en refusant ce que le communisme avait de meilleur: la tradition antifasciste, le respect des morts et le souvenir des victimes du fascisme qu'on peut construire une nation libre. La juste colère se fait sarcastique devant le cynisme ${ }^{8}$ des nouveaux maîtres qui n'hésitent pas à transformer en une discothèque un ancien camp de concentration où des milliers d'enfants juifs ont été rassemblés en vue de leur extermination ${ }^{9}$. En un rapprochement saisissant, l'auteur compare " les orteils repus " des danseurs aux orteils déchiquetés par la grenade qui arracha leurs jambes aux frères jumeaux de la narratrice. Marica reste le témoin extérieur qui assiste désarmé au désastre, à la disparition de ce pays dont les parfums ensoleillés nourrissaient encore l'imaginaire longtemps après l'émigration. À la place de la terre natale, du pays des oliviers sacrés et des cyprès, symboles de paix et d'accueil, avaient surgi la "patrie ", l'arrogance, le dogmatisme et l'esprit mercantile. Les paysages féeriques, mythiques étaient devenus des slogans publicitaires: désormais, la mer, les montagnes se vendaient à l'encan. Au communisme athée, on opposait un christianisme arrogant. Enfin, le commerce et le tourisme ne peuvent en aucun cas constituer les seules alternatives au socialisme.

11 Le thème de l'enfance assassinée et de l'innocence violée se prolonge ainsi d'un roman à l'autre, l'un évoquant le camp de concentration, l'autre la monstruosité des bourreaux. Tandis que, dans le premier consacré à La Mémoire des Libellules, Nadeshda découvre que son père, grand admirateur d'Hitler et de Staline, tuait les enfants comme il le faisait avec les libellules, Arjeta frémit d'horreur face à la profanation des lieux de la mémoire. L'amitié des deux femmes prend une signification très particulière quand on se rappelle l'expérience menée en Allemagne pour surmonter les traumatismes du national-socialisme. Instaurée dans les années 1950, cette thérapeutique consiste en effet à mettre en contact des descendants de bourreaux avec ceux de victimes pour 
qu'ensemble, ils puissent surmonter la souffrance et le poids d'un passé sans cela insupportable. Le lecteur pense aussi au massacre des innocents, ce meurtre de tous les enfants de moins de deux ans ordonné par Hérode après la naissance de Jésus. Les enfants, proies faciles car sans défenses, sont comme des libellules, d'une beauté encore évanescente et pure, légers et innocents. Comme elles, ils ont la limpidité de la lumière qui se reflète sur leurs ailes transparentes. Dans la poésie persane, le papillon est un symbole de l'âme... toutes ces libellules crucifiées par le père fasciste de Nadeshda ne sont-elles point les âmes de ces enfants sacrifiés sur l'autel de la folie raciste et du mythe de l'unité?

12 À la tragédie humaine s'ajoute la tragédie culturelle et linguistique d'une nation vouée à l'extinction. Igor Stickš établit lui aussi un lien entre la Seconde Guerre mondiale et celle de Yougoslavie, quand il éclaire en particulier le siège de Sarajevo en 1992 dans une perspective européenne. Est-ce un hasard si l'héroïne de Kirschholz porte le même nom que celle de Archive der Nacht, Filipo faisant écho à Filipovic? À l'instar d'Igor Stickš, Marica Bodrožić pose la question d'Homo Faber sur les limites du savoir rationnel qui ne parvient toujours pas à empêcher les violences, la barbarie et la férocité. L'homme moderne croit au progrès, il se croit maître de son destin et, dans la continuité des Lumières, pense pouvoir vaincre l'obscurantisme religieux ou national par la science et les mathématiques. Mais il ne peut empêcher qu'un sniper fou assassine une femme faisant son marché ${ }^{10}$. Physicienne, Nadeshda ne trouve pas dans sa spécialité la réponse à la question angoissante du sens. Comment peut-on vivre après tant d'horreurs? Elle ne la trouve d'ailleurs pas non plus dans la Bible. Peut-on y voir la marque du destin? ou la volonté d'un dieu vengeur? Ou avec les surréalistes le « hasard objectif »? Comme Mallarmé, elle compare la vie humaine à une partie de dés. L'homme joue aux dés, il gagne ou il perd. Mais, on pourrait rapprocher la remarque de Nadeshda : "Ich würfle », à celle de Mallarmé quand il affirmait : "Penser, c'est jouer aux dés.». Ainsi, cette expression décrirait-elle seulement la pensée, ou plus précisément la conscience, la prise de conscience de la narratrice.

\section{Support de l'identité perdue : la photographie}

13 Arjeta Filipo resserre le lien avec le passé yougoslave de sa famille, grâce à la table héritée de sa grand-mère. Le merisier blond parfume la pièce, les photos étalées sur le bois si fin, font surgir peu à peu les scènes du passé, et avec elles surgit le sens qui ordonne le chaos de la vie au présent. Les moments éphémères fixés sur la pellicule prennent une signification, inconnue au moment même de la photographie, pour constituer le principal support identitaire. Arjeta vide sur la table les sacs en plastique dans lesquels sa mère a jeté les clichés sans ordre ni système, détruisant la chronologie que seul le souvenir plus ou moins précis se charge de rétablir. Au contact du bois chaud, les images reprennent vie et retrouvent peu à peu leur place dans le temps permettant à la narratrice de recomposer le tableau et de reconstruire le moi éparpillé sur la table sous la forme de ces photos jaunies... Arjeta rassemble peu à peu les éléments dispersés de la mosaïque, pour reconstruire son identité disloquée. Écrire un roman, c'est en quelque sorte faire l'inventaire du passé, du « moi », retrouver le moi dissimulé sous la couche épaisse des non-dits, des mensonges par omission et de l'autoaveuglement. 

présent meurtrier avait ôté tout leur sens à ses photos souvenirs de moments heureux irrémédiablement révolus. Incapable cependant de les jeter et donc de condamner à l'oubli absolu les moments perdus, auxquels elle n'ose plus croire, elle confie à sa fille le soin de rétablir l'ordre dans le désordre des photos jetées pêle-mêle dans les sacs de la droguerie. Pourtant ce désordre même fait surgir la vie et permet de la retrouver dans sa multiplicité.

À ce comportement auto-destructeur, Marica Bodrožić oppose le voyeurisme compulsif d'Arik. Arik range sagement ses photos dans des albums, comme s'il voulait retenir chaque seconde de la vie, les contraindre dans des catalogues. Il photographie chaque mouvement, chaque situation, chaque détail du corps, une main, une joue, une oreille. Les photos sont bien rangées dans des albums eux-mêmes enfermés dans des armoires qui transforment l'appartement situé au cœur historique de Paris, la place Dauphine, un des lieux magiques du Paris d'André Breton, en une photothèque, une bibliothèque... comme s'il pouvait empêcher le temps de s'écouler, le fixer. Avec ses instantanés, Arik tente de s'approprier la réalité qu'il est incapable de vivre. Focalisée sur les détails du corps de la femme convoitée : une main, un pied, une joue, l'image exprime le monde de façon fragmentaire... Arjeta est saisie d'horreur en découvrant cet archivage de la réalité, de sa réalité. Sa vie ne lui appartient plus. Elle est devenue un objet d'observation. effet une tentative vaine d'appropriation du réel. Elles retiennent tous les instants de la vie d'Arjeta. Elles la poursuivent, l'espionnent. Mais loin de permettre la rencontre, l'échange et la fusion, elles séparent. Incapable de vivre, inapte au bonheur, Arik veut dans sa folie découper, agencer, organiser le réel, et, ce faisant, il le perd. Comme le père de Nadeshda qui tuait les libellules pour les classer dans ses albums, Arik détruit la vie en photographiant ses moments les plus intimes en lui préférant la recherche esthétique, par son désir de tout transformer en art. Ici l'art et la vie s'opposent. La photographie qui se veut être une reproduction exacte de la vie, une copie réaliste, est en réalité une falsification mortifère !

\section{La libération et l'amour fou}

L'entrée d'Ilja dans la vie de Nadeshda, et d'Arik dans celle d'Arjeta, signifient pour les deux femmes la découverte de soi, une intensification et une poétisation du moi correspondant paradoxalement à un abandon de soi. Et c'est seulement avec la disparition de l'amant que la quête ou plutôt la reconquête commence. Dans La Mémoire des Libellules, la narratrice, en laquelle on pourrait reconnaître l'auteur, avoue son intérêt mêlé d'admiration et de répulsion pour Nadja d'André Breton. Comme Nadja, le roman est le récit d'un amour exclusif, d'un « amour fou ». Nadja en russe est le début du mot "espérance » et Nadeshda est un prénom russe signifiant "espoir ». Dès le deuxième chapitre, le lecteur est entrâné sur les traces d'André Breton et du surréalisme. Tout comme Nadja dans le récit d'André Breton, la narratrice dit s'être attribué elle-même ce nom qui n'est pas celui choisi par ses parents. Avec Breton, elle compare l'amant à un paysage inconnu dont elle explore les secrets, les merveilles cachées. C'est à Paris que Nadeshda rencontre Ilja. Et cette rencontre bouleverse sa vie en lui faisant découvrir son corps. Les lieux de l'amour entre Arjeta et Arik sont ceux de 
Nadja: la Seine, la place Dauphine que Breton compare au sexe de Paris ${ }^{11}$... Avec Guillaume Apollinaire, elle se rend sur le pont Mirabeau, et c'est sur ce pont qu'Arik la possède. C'est la rue de Paris qui lui révèle cette passion soudaine, envahissante, exclusive, bouleversante, voluptueuse et charnelle. Nadeshda ignore le calcul, le repos, la raison lénifiante. Son amour pour Ilja est un attachement et un don total de toute sa personne, gratuit car sans espoir d'être partagé en retour. Il ne tolère pas la médiocrité et ne s'enferme pas dans les liens du mariage bien qu'il accepte de donner la vie à un enfant, Ezra, dont le nom signifie «aide ». L'enfant de l'amour est une aide, un guide permettant d'accéder à la vérité. L'amant reste un inconnu, un mystère ${ }^{12}$ insondable. La rencontre avec Ilja ouvre la voie ${ }^{13}$ à un parcours initiatique permettant à Nadeshda de répondre à la question qu'elle pose avec André Breton en incipit : «Qui suis-je ${ }^{14}$ ? ॥ Il rend à la vie le sens qu'elle a perdue dans la guerre dont il constitue le pôle opposé, le dernier espoir. Il est un service divin, sacré ${ }^{15}$. Et quand Nadeshda cherche sa confirmation dans la Bible, il est au plein sens du terme une révélation, il devient presque mystique.

Cependant, la perspective adoptée n'est plus celle de l'homme, André Breton, mais celle de Nadja, la femme si follement aimée avant d'être abandonnée. Nadja avait su, selon les principes du surréalisme, dépasser les limites de la raison au prix de sa vie, et pourtant, Breton, sans le moindre regret, la condamne au silence absolu derrière les murs d'un hôpital. C'est cette femme trahie qui prend la parole. Le roman de Marica Bodrožić donne la version féminine de "l'amour fou ", pour en réfuter la vision trop masculine. Nadeshda n'accepte pas cette déloyauté du poète, car l'amour est «fou » sans doute, par son exclusivité, son refus des normes, du bon goût, de la logique, de la morale et de la prudence, par son désir d'absolu, mais cette folie ne ressemble en rien à celle des hospices qui signifie la mort sociale, cet obscurcissement de la conscience, la nuit de l'esprit si bien exprimée par le mot allemand de Umnachtung. Tout au contraire, le voyage de Nadeshda la conduit non à perdre la conscience mais bien à la retrouver, à se reconstruire elle-même, à comprendre la prudence lâche de l'homme qui, dit-elle, lui donne assez d'amour pour se l'attacher, mais pas assez pour renoncer à son confort bourgeois ${ }^{16}$. L'amour fou défini par Breton inclut le merveilleux, l'imagination, le rêve. Et ce rêve ne peut être assassiné par la médecine. Le roman est en fait une quête sur le moi, et sur la passion amoureuse, sur la liberté et la poésie. Les libellules n'incarnentelles pas la légèreté, la transparence des fées ? Et leur assassinat par le père inconnu ne serait-il point la vraie folie des hommes incapables d'aimer, inaptes à apprécier la beauté, toujours prêts à tuer ce qui bouge, ce qui sent, ce qui vit, aveugles qui opposent la « raison" des règles et des lois à la multiplicité foisonnante de la vie ? Tout à l'opposé, les libellules se posent naturellement sur les épaules de Nadeshda signifiant son intimité avec le monde des fées.

L'amour d'Ilja lui permet de naître une seconde fois malgré les crimes monstrueux des fascistes et des communistes. À l'amour qui irrigue tout de sa vigueur et de sa générosité, le roman oppose la volonté mécanique, consumériste de jouir sans limite de plaisirs vains, de s'enivrer, de s'étourdir de musiques violentes pour faire taire la mauvaise conscience. Pour contrer cette vision matérialiste de la vie, Marica Bodrožić propose l'amour fou et la poésie des surréalistes. Nadeshda incarne l'espoir d'une renaissance par le don gratuit de soi dans l'amour. La passion de Nadja ne nie ni le crime ni la guerre, et celui d'Arjeta et de Nadeshda surgit pendant le siège de Sarajevo. Mais contrairement aux danseurs de la discothèque, ni Nadeshda ni Arjeta ne nient les 
atrocités dont elles ont été les témoins. Renaître, accepter la vie malgré la guerre, n'est pas un oubli mais une force. Quand une réalité meurtrière s'attache à tout détruire, quand le monde tout autour s'écroule et que Sarajevo est réduite à un amas de pierres, seul l'amour permet de survivre. Lui seul est, comme dans le poème de Lucrèce, source de vie et de renaissance. L'amour renait sans cesse sur les décombres encore fumantes de la civilisation ${ }^{17}$; unique, il est intransigeant malgré le départ de l'amant, malgré la trahison de celui qui préfère le "havre» commode du mariage à la passion déstabilisatrice qu'il ne s'accorde qu'au hasard des congrès et des colloques. Nadeshda accepte la trahison d'Ilja, Arjeta surmonte le choc de la macabre découverte des albums au nom de cet unique instant, de la magie d'un été. Car l'absolu ne signifie point la possession exclusive de l'autre. Ce qui importe, c'est l'expérience de l'absolu.

L'amour n'est pas source de bonheur, comme l'est le mariage pour Ilja qui ne quittera jamais sa femme. Don de soi, il est service et non pas asservissement; libre, il ne se soumet à aucune loi ; mystique, il refuse les dogmes de la religion, ses lois, ses règles. Marica Bodrožić réinterprète la parabole kafkaïenne "Vor dem Gesetz» et fait du gardien de la porte un dictateur féroce qui emprisonne l'amour dans un livre de lois ${ }^{18}$. C'est que l'amour est d'abord désir, désir brûlant de l'inconnu, du mystère qui se cache derrière la porte de la Loi. Nadeshda unit alors l'image du coffret et de la clef utilisée par André Breton ${ }^{19}$ à celle de la porte kafkaïenne: la clef du coffret réside dans la réalisation du véritable amour. Pourtant, la clef que l'amant lui a donnée ne sert qu'à ouvrir son propre cœur, à le lui livrer sans défense, tandis que le sien se ferme. Ilja, insensible, a certes ouvert la maison de son cœur, mais il refuse d'entrer. Toute la vie n'est plus qu'une attente infinie, langoureuse et cruelle, attente vaine et non stérile. Ce que Nadeshda découvre dans cette longue attente, c'est que la volupté, et non la rationalité scientifique, est la première source de la connaissance. Elle se passionne pour Georges Bataille et, avec lui, doit réunir Eros et Thanatos. Car la vraie réponse à la quête initiatique déclenchée par Ilja, on la trouve plutôt chez Georges Bataille qui donnait à l'érotisme le profil de la dissolution que chez André Breton, qui n'a lui-même jamais été capable de vivre l'idéal défendu dans Nadja.

21 Des ruines de leurs malheureuses amours surgit une autre affinité, l'amitié des deux femmes élevant ensemble l'enfant fruit de la passion. Les prénoms sont des signes : le nom albanais d'Arjeta se traduit par «l'or de la vie », le prénom russe Nadeshda par «l'espérance ». Nadeshda souhaiterait s'appeler Sam, en référence au roman du dernier auteur yougoslave Danilo Kiš, Jardin, cendre ${ }^{20}$ et à son personnage Edouard Sam, écrivain anarchiste, ermite pacifiste, poète et philosophe aux pieds plats. Arjeta donne «l'or de la vie » à Nadeshda qui à son tour lui rend «l'espoir ». Ezra, leur vient en « aide » pour continuer à vivre. Si le nom de Nadeshda rappelle l'espoir de l'amour, celui d'Arjeta suggère le rêve alchimiste d'André Breton qui cherchait "l'or du temps ", et a même fait graver cette phrase en épitaphe sur sa tombe. Les trois noms réunissent aussi trois nations, trois identités, un peu comme le faisait la Yougoslavie naguère. Leur trio dépasse donc le bonheur individuel pour symboliser celui des peuples et des générations réconciliés; enfants de victimes, enfants de bourreaux se retrouvent pour créer un monde plus généreux, sans illusions mais nourri de rêves.

Pour conclure on reconnaît dans les romans de Marica Bodrožić, certes quelques éléments autobiographiques et son évocation de la diaspora yougoslave à Paris correspond sans doute à des expériences vécues. Les lieux évoqués, elle les a découverts lors de ses divers voyages. Il est vrai qu'elle est venue s'installer à Berlin après un 
séjour d'études à Paris. Pourtant, malgré l'emploi de la première personne et l'introspection minutieuse, malgré la relation au style direct, ni Kirchholz ni Libellen ne sont des romans autobiographiques. Et s'ils se concentrent sur l'évocation d'une passion magique et bouleversante, c'est pour en montrer la fonction d'initiation. Cette passion nourrie par le désir, par l'attente, par la disparition de l'objet aimé, l'un partant pour les États-Unis retrouver son foyer, l'autre mourant soudainement, n'est pas destructrice. Elle est un voyage initiatique dans les paysages intérieurs, elle ouvre et élargit la conscience, poétise le monde, elle est la seule source de vie contre le dogmatisme destructeur des idéologues. Pourtant l'homme à qui s'adresse cet amour désintéressé, absolu et presque sacrificiel, ne partage point le trouble déstabilisateur qu'il a lui-même provoqué par ses fausses promesses. Et l'on peut imaginer que Nadeshda fait à André Breton le reproche qu'il a lui-même adressé à Nadja : celui de l'indifférence et de l'incompréhension. On pourrait alors concevoir le roman Das Gedächtnis der Libellen comme une réfutation de Nadja d'André Breton. Au-delà de cette réfutation, l'amitié des deux femmes fait surgir un autre espoir : l'or de la vie, ce que la vie a de plus précieux, de plus beau, peut se trouver dans l'amitié, dans le désir, dans ce mouvement qui nous entraîne toujours vers un ailleurs.

\section{NOTES}

1. Marie-Hélène Quéval, "Marica Bodrožić, écrire dans une langue étrangère », Une nouvelle littérature allemande: écrivains germanophones venus d'ailleurs in: Dirk Weissmann (dir.), "Littérature et migration : écrivains germanophones venus d'ailleurs ", Allemagne d'aujourd'hui, n¹97, 3/2011 (juillet-septembre).

2. Marica Bodrozic, Das Geheimnis der Libellen, München 2010, Taschenbuchausgabe 2012, btb p. 25 (sigle : Libellen).

3. Marica Bodrozic, Kirschholz und alte Gefühle, München Luchterhand 2012 (sigle Kirschholz).

4. «Arjetas Singular ist an sich ein großer Plural, sie ist eines jener typischen jugoslawischen Kinder, die nach dem Krieg wie kleine Vogelkinder in der Heimatlosigkeit der Luft fliegen lernen. ", Libellen, p. 25.

5. Igor Stickš, Die Archive der Nacht Ein Holzstuhl für Elias oder das Manuskript des Richard Richter, aus dem Kroatischen von Marica Bodrozic, Berlin Claasen, 2006 (sigle : Archive).

6. «Sie hatten nur eines im Sinn: Die eigene Sprache zu erhalten, sie zu lieben und vor fremden Einflüssen zu beschützen. Das war jetzt ihre wichtigste Aufgabe. Weg mit der Literatur der Feinde! Weg mit dem anderen Alphabet. - Sie gehörten doch nicht zu Moskau und den Russen!", Kirschholz, p. 176-177.

7. «Aber wie verwundet sie innerlich nach all den Jahren immer noch war, hörte ich jetzt an der Art, wie sie über alles erzählte, wie sie sich jeden Satz von Mateos Verein gemerkt hatte und in seiner Sprache wie in ein offenes Messer gerannt war. Sie war verwundet worden und eingezäunt von dieser Sprache, die keine Gnade kannte. », Kirschholz, p. 183.

8. « Das Hier. Das Jetzt. Das richtige Leben. Einst bekamen in genau diesen Räumen die kleinen Gefangenen ihr Essen mit Natriumhydroxid vermischt. Hier in den gleichen Räumen, sagte meine Mutter, in denen ihr Appetit tödlich war, tanzen heute satte, glückliche, schöne, moderne Jugendliche in bunten Converse Chucks zu betörend schnellen Beats. Wenn sie am frühen 
Morgen nach Hause wankten, lauschten sie dem Singen der vielen Nachtigallen, der Tau auf dem Gras grüßt ihre durchgetanzten Zehen. Es ist ein wohliges Leben, das sie haben - ein sattes Leben im Frieden, voller Möglichkeiten, offen für die Zukunft, für jedes Abenteuer. » Kirschholz, p. 185.

9. Kirschholz, p. 184.

10. «Einmal, das habe Mateo ihr selbst in Großmutters Haus erzählt, hatte er einen Mann neben sich im Graben weinen sehen und ihn von ganzem Herzen dafür verachtet. Auch dann, als dieser ihm erzählte, dass seine Frau auf dem Weg zum Markt erschossen worden war, erschossen von Leuten, die zufällig zum ersten Mal eine Waffe benutzten, herumprobierten und aus Spaß ins Nichts zielten. Zufällig ging sie, gerade in Gedanken versunken, durch dieses Nichts in Richtung Marktplatz, um für ihre Kinder Besorgungen zu machen. Das hatte ihm seine elfjährige Tochter geschrieben. », Kirschholz, p. 176.

11. "C'est à ne pouvoir s'y méprendre, le sexe de Paris qui se dessine sous ces ombrages. » (Pléiade, III, 893).

12. «Wir leben um das Geheimnis herum, zergliedern es, machen Bilder aus ihm, Metaphern, Umwege, Falten und Krankheiten. Aber das Geheimnis bleibt. » Libellen, p. 21.

13. Libellen, p. 23.

14. "Wer bin ich? Ich heisse Nadeshda. Meinen Namen habe ich nicht von Nadeshda Mandelstam. Und um es klar zu machen, das ist noch wichtiger, von meinen Eltern habe ich den Namen nicht bekommen. Meine Eltern haben mir einen ganz anderen Namen gegeben. Es ist ein Name, der gar nicht zu mir passt. Ich selbst habe den neuen Namen für mich gefunden, damit ich diese Geschichte erzählen kann. » Libellen, p. 19.

15. «In der Liebe dient man nur. », Libellen, p. 23.

16. «Ilja ist ein Mann. Er wird natürlich nie in ein Irrenhaus wie Nadja landen, dazu weiss er zu viel über seine eigene Gefährdung. Er ist nie wie Nadja für mich gewesen, weil ich seine Nadja war. Noch immer stecke ich im bitteren Teil dieser Wahrheit selbst... ", Libellen, p. 107.

17. « Diese Stadt ist keine Wildnis ! Sie gehört zur Zivilisation! », Kirschholz, p. 26.

18. Libellen, p. 23.

19. Pléiade II, 270.

20. Danilo Kis, Bašta, pepeo (1965), Jardin, cendre, traduction française de Jean Descat, Gallimard, 1971.

\section{RÉSUMÉS}

Originaire de Croatie, Marica Bodrožić avait à peine dix ans lorsqu'en 1983, elle quitta son pays natal pour rejoindre ses parents en Allemagne. Elle ressentit un amour presque immédiat pour la langue allemande qui, certes signifiait le renoncement à l'identité originelle de l'enfant mais lui permit aussi d'acquérir une identité nouvelle associant harmonieusement la sensibilité méditerranéenne à l'exigence de rigueur imposée par l'apprentissage d'une langue étrangère. Si dans une première période, Marica Bodrožić écrit surtout des poèmes et des contes philosophiques, elle s'est depuis quelques années consacrée avec succès au genre romanesque. Selon les nouvelles théories du roman postmoderne, elle échappe au réalisme historique ou biographique pour lui préférer le réalisme poétique. Car si la dislocation de la Yougoslavie et la tragédie de Sarajevo constituent la trame de fond de ces deux derniers romans, La Mémoire des Libellules et Le Merisier et les sentiments anciens, ces drames n'en sont pas le sujet central. Marica 
Bodrožić pratique avec talent une écriture fondée sur le collage, la citation et l'intertextualité. Les deux romans constituent en réalité la version féminine des récits d'André Breton : Nadja et L'amour fou. C'est ce dialogue avec le poète surréaliste français que l'article analyse.

Born in Croatia, Marica Bodrožić was only nine when, in 1983, she left home to join her parents in Germany. She almost immediately fell in love with the German language and as a result, though she lost her childhood identity, she acquired a new identity that harmoniously combined her Mediterranean sensibility with the intellectual rigour needed to learn a new language. She began writing poems and philosophical short stories, but over the last few years Marica Bodrožić has become a successful novelist. Marked by postmodern theories of fiction, her brand of realism is poetic, rather than historical or biographical. The break-up of Yugoslavia and tragic events in Sarajevo provide the setting but not the main subject for her two most recent novels, Das Gedächtnis der Libellen (The Memory of Dragonflies) (2010) and Kirschholz und alte Gefühle (The Cherrytree and Old Feelings) (2012). Using collage, quotations and intertextual references, these two novels are in fact her female response to André Breton's books Nadja and L'Amour fou (Mad Love). This article explores Marica Bodrožić's literary dialogue with the French surrealist poet.

Marica Bodrožić stammt aus Croatien und ist erst im zehnten Lebensjahr zu ihren Eltern nach Deutschland gezogen, in einem Alter, wo man ganz bewusst, eine erste Sprache aufgeben muss, um eine neue zu erlernen. Diese Frage nach der Sprache und der Identität, die sie nur als „Plural“ versteht, wird in ihren Werken immer wieder thematisiert, auch wenn sie kein Vorbild für eine gelungene Integration sein will. Ihre Gedichte vereinigen somit die mediterrane Sensibilität mit der Genauigkeit der deutschen Sprache. Nach einer ersten Schaffensperiode, wo sie hauptsächlich Gedichte und philosophische Erzählungen veröffentlicht hat, hat sie sich seit einigen Jahren zur Romanschriftstellerin entwickelt. Dieser Artikel untersucht ihre letzten zwei Romane: Das Gedächtnis der Libellen (2010) und Kirschholz und alte Gefühle (2012). Indem sie die Technik der Collage mit mehr oder weniger sichtbaren Zitaten verbindet, praktiziert sie die für den postmodernen Roman typische Intertextualität. Geschichtliche Ereignisse wie das Auseinanderfallen Jugoslawiens und die Belagerung Sarajevos bilden den Hintergrund. Jedoch geht es hauptsächlich hier nicht um eine realistische Schilderung historischer Ereignisse, sondern viel eher um die Darstellung einer in sich geschlossenen Subjektivität und einer gebrochenen Persönlichkeit. Beide Romane setzen sich tatsächlich vielmehr mit André Bretons Nadja auseinander als mit der Geschichte Jugoslawiens. Diese intertextuelle Beziehung zu Nadja ist Gegenstand der Untersuchung.

\section{AUTEUR}

\section{MARIE-HÉLÈNE QUÉVAL}

Université du Maine 